Research Paper

\title{
Calpain-2 Enhances Non-Small Cell Lung Cancer Progression and Chemoresistance to Paclitaxel via EGFR-pAKT Pathway
} \author{
Ronghua $\mathrm{Liu}^{2 \bowtie}, \mathrm{Di} \mathrm{Ge}^{1 \bowtie}$ \\ 1. Department of Thoracic Surgery, Zhongshan Hospital, Fudan University, Shanghai, P. R. China. \\ 2. Department of Immunology, Fudan University, Shanghai, P.R. China. \\ *These two authors contributed equally to this work.
}

Fengkai $\mathrm{Xu}^{1 *}$, Jie $\mathrm{Gu}^{1 *}$, Chunlai Lu${ }^{1}$, Wei Mao1, Lin Wang1, Qiaoliang Zhu ${ }^{1}$, Zhonghe Liu1, Yiwei Chu²,

$\triangle$ Corresponding authors: Di Ge, M.D., Ph.D., Department of Thoracic Surgery, Zhongshan Hospital, Fudan University, No. 180 Fenglin Road, Shanghai, China, Tel. / fax: +86-021 64041990, E-mail address: ge.di@zs-hospital.sh.cn. and Ronghua Liu, Ph.D., Key Laboratory of Medical Epigenetics and Metabolism, Institute of Biomedical Sciences, and Shanghai Fifth People's Hospital, Fudan University, No. 138 Yixueyuan Road, Shanghai, China, Tel. /fax: +86-021-54237362, E-mail address: 10211010035@fudan.edu.cn.

(c) Ivyspring International Publisher. This is an open access article distributed under the terms of the Creative Commons Attribution (CC BY-NC) license (https://creativecommons.org/licenses/by-nc/4.0/). See http://ivyspring.com/terms for full terms and conditions.

Received: 2018.07.28; Accepted: 2018.10.20; Published: 2019.01.06

\begin{abstract}
Lung cancer is one of the most frequent malignant tumors, with the top morbidity and mortality, in China. Calpain family regulates cellular processes including migration and invasion. However, the role of Calpain-2 in non-small cell lung cancer (NSCLC) remains unclear. This study aims to explore the bio-function of Calpain-2 on NSCLC and chemoresistance to paclitaxel. In this study, Immunohistochemistry, RT-qPCR and Western blot were performed to detect the Calpain-2 expression and related pathway protein in NSCLC. The Kaplan-Meier product limit estimator and Cox regression were conducted for survival analysis. CCK-8, Transwell, colony-formation, apoptosis and tumor xenograft assays were performed to analyze tumor-promoting role of Calpain-2, and the chemoresistance to paclitaxel. Our data showed that Calpain-2 was up-regulated in NSCLC. Notably, Calpain-2 level positively correlated with differentiation grade and negatively correlated with the 5-year overall survival, which served as an independent prognostic predictor. Knockdown of Calpain-2 inhibited cell proliferation and migration, while promoted apoptosis in vitro. In vivo, Calpain-2-knockdowned cells formed smaller subcutaneous tumors. Meanwhile, knockdown of Calpain-2 down-regulated EGFR and PAKT expression, which weakened the chemoresistance of NSCLC cells to paclitaxel by suppressing cell proliferation and inducing apoptosis, and even enhanced the paclitaxel-mediated downregulation of EGFR and pAKT level. To conclude, Calpain-2 might activate EGFR/pAKT pathway to promote NSCLC progression and contributes to the chemoresistance to paclitaxel, which might be a therapeutic target to prevent or postpone the progression of NSCLC.
\end{abstract}

Key words: Calpain-2, non-small cell lung cancer, paclitaxel, chemoresistance, prognosis

\section{Introduction}

Lung cancer is one of the most frequent malignant tumors all over the world. In China, lung cancer occupies the top morbidity and mortality presently. Non-small cell lung cancer (NSCLC) accounts for nearly $85 \%$ of all types of lung cancer [1]. Benefit from the wide use of targeted therapy, lung cancer patients' prognosis has been improved during the last ten years [2]. However, for the high tendency to recurrence and metastasis, together with the fact that targeted therapy might lead to drug-resistance with time, the 5-year survival rate of lung cancer patients still remains low [3]. Therefore, it is quite 
urgent to discover novel deeper mechanism for the carcinogenesis and progression of lung cancer, which would contribute to the development of novel targeted therapy.

Calpains are a family of calcium-dependent intracellular cysteine proteases, among which $\mu$ Calpain and m-Calpain are two most extensively studied members [4]. In the analysis of the structure, both $\mu$-Calpain and $\mathrm{m}$-Calpain were composed of distinct large catalytic subunits of $80 \mathrm{kDa}$, Calpain-1 and Calpain-2, respectively, and a small common regulatory subunit of $28 \mathrm{kDa}$ encoded by Calpain-s1 (CAPN4) [5]. Calpains have been reported to regulate cellular processes including migration and invasion by cleaving cytoskeletal proteins [4], which might contribute to the metastasis, even progression of malignant tumors. In our previous study, we have demonstrated that CAPN4 could promote NSCLC proliferation and invasion with the activation of matrix metalloproteinase 2 [6]. Considering multiple studies demonstrated that Calpain-2 is dysregulated in different tumors, including colorectal adenocarcinomas, glioblastoma, prostate cancer, renal cell carcinoma, etc [7-10], and no related study has been involved in NSCLC, we intend to explore whether Calpain-2 could make a difference in the carcinogenesis and progression of lung cancer. Furthermore, Calpain-2 has also been reported to weaken the response to platinum based chemotherapy in ovarian cancer [11]. Meanwhile, Calpain family participates in the paclitaxel-induced peripheral neuropathy [12]. Thus, we also tried to analyze the association between Calpain-2 and resistance to paclitaxel (PTX), another first-line chemotherapeutics for NSCLC.

In this study, we aimed to investigate the bio-functional and prognostic role of Calpain-2 in NSCLC tissues and cells. Our data demonstrated that Calpain-2 was up-regulated in NSCLC tissues, and high Calpain-2 level negatively associated with the 5 -year survival of patients. We also revealed that Calpain-2 could promote NSCLC cells proliferation and migration, while inhibit apoptosis ability, even enhance the resistance to PTX possibly via EGFR-pAKT signaling pathway, which might provide a novel therapeutic strategy to prevent or postpone the progression of NSCLC.

\section{Materials and Methods}

\section{Tissue microarray, immunohistochemistry and follow-up}

Tissue microarrays and immunohistochemistry were performed as previously described [13].The Calpain-2 antibody (Abcam 75994, Cambridge, UK) was used to detect Calpain-2 expression in tissue miscroarrays. Each section was semi-quantitatively analyzed for the extent of immunoreactions and staining intensity, with two experienced pathologists who were unclear of the content or the purpose of the study. The extent of immunoreactions was scored as: 0 (0\% immunoreactive cells), 1 (1-10 \% immunoreactive cells), 2 (11-50\% immunoreactive cells) or 3 (>50\% immunoreactive cells). The staining intensity was scored as: 0 (negative), 1 (weak, +), 2 (intermediate, ++ ), or 3 (strong, +++ ). The total immunoreaction score was calculated as the sum of both two factors above. All the samples were classified into negative (0), weak (1-2), moderate (3-4) and strong (5-6) staining groups, respectively. For the purpose of statistical analysis, negative and weak cases were incorporated into the Calpain-2 ${ }^{\text {low }}$ subgroup, and moderate and strong cases were incorporated into Calpain-2high ones. Another six pairs of frozen human NSCLC tissues and matched para-cancerous tissues were obtained from six patients at Zhongshan Hospital in 2014. The para-cancerous tissues were the normal tissues within $3 \mathrm{~cm}$ of cancer tissue.

In this study, patients who received neo-adjuvant therapy were precluded in advance. Patients whose pTNM stage were over stage II received post-operative chemotherapy. Patients with mediastinal lymph node metastasis received post-operative radiotherapy. For the follow-up, each patient was required to take chest $\mathrm{CT}$, abdominal ultrasonography and serum tumor markers every three month during the first year after surgery. Then, the follow-up period was extended to twice a year. At the each anniversary of surgery, Brain MRI and full body bone scan were applied. The follow-up was ended in July, 2010.The overall survival (OS) period was defined as the time span from the surgery date to the death date. The study has got the approval of the ethics committee on human research of Zhongshan Hospital, Fudan University.

\section{Cell lines culture, transfection and Western blot assay}

The human NSCLC cell line (A549, H1299, H1395, 95C and 95D) and human normal bronchiolar epithelium cell line (HBE) were purchased from the Chinese Academy of Sciences. A549, H1299, H13 95, 95C, 95D and HBE were cultured in RPMI-1640, and A549 was cultured in DMEM. Both two medium were supplemented with $10 \%$ fetal bovine serum and $100 \mathrm{IU} / \mathrm{ml}$ penicillin/streptomycin in a humidified incubator, under $95 \%$ air and $5 \% \mathrm{CO} 2$ at $37^{\circ} \mathrm{C}$.

Lipofectamine 2000 (Invitrogen, Carlsbad, USA) was applied for transient transfection. Predesigned siRNA duplexes were purchased from Genomeditech 
Company. The sequences of siRNA-Calpain-2-1 are 5'- CCGAGGAGGUUGAAAGUAA -3' (F) and 3'GGCUCCUCCAACUUUCAUU-5' (R). The sequences of siRNA-Calpain-2-2 are 5' - CGAGAAUACUGGAA CAAUA-3' (F) and 3'- GCUCUUAUGACCUUGUUA U-5' (R). The sequences of siRNA-Calpain-2-3 are 5'-GCUCAGACACCUUCAUCAA' (F) and 3'- CGAG UCUGUGGAAGUAGUU-5' (R). The knockdown group was cells transfected with siRNA-Calpain-2 sequence. The normal control group was cells transfected with a non-targeted scramble sequence.

The transfection and Western blot assay were performed as previously described [13]. PTX-treated subgroups were culture in the medium added with 20nM PTX for 24 hours, following transfection for 24 hours. The protein extraction was performed at 48 hours after transfection. The Calpain-2 antibody (Abcam 75994, Cambridge, UK) was used to detect the Calpain-2 expression. And the other antibodies were listed in Table S1.

\section{RNA extraction and RT-qPCR analysis}

The total RNA extraction and RT-qPCR analysis were performed as previously described [13]. The primers of Calpain-2 are 5'-CAGCCATTGCCTCCCT CAC-3' (F) and 5'-TCCACCCACTCGCCGTATT-3' (R). The primers of $\beta$-actin are $5^{\prime}$-TGACGTGGACATC CGCAAAG-3' (F) and 5'-CTGGAAGGTGGACAGCG AGG-3' (R).

\section{CCK-8 assay and Transwell assay}

CCK-8 assay was performed as previously described [14]. After transfection, the OD values were detected at certain time thereafter, respectively. For the analysis of PTX function, at 24 hours after transfection, cells were treated with PTX with different concentration for another 24 hours. And then the OD values were detected. Transwell assay was also performed as previously described [15]. Each experiment was repeated 3 times. The sensitivity of each type of cell to PTX was also evaluated with CCK-8, presented as the relative cell viability (\%). The concentration of PTX at which $50 \%$ of cells survived presents the IC 50 value.

\section{FACS analysis of apoptosis}

Apoptosis assay was performed with Annexin V-FITC/PI Apoptosis Detection Kit (YEASEN, Shanghai, $\mathrm{CN}$ ). At 48 hours after transfection, cells of each subgroup were collected and washed three times with PBS, then suspended in $100 \mu 1 \times$ binding buffer, added with the 5ul Annexin V-FITC and 10 Propidium Iodide (PI) incubated at room temperature for 15 minutes in the dark. The cells stained with Annexin V and PI were detected with flow cytometry on a
FACScan (BD Biosciences, NJ, USA) in the corresponding procedures. For the analysis of PTX function, at 24 hours after transfection, cells were treated with 20nM PTX for another 24 hours and then collected for the analysis of apoptosis. Each experiment was repeated 3 times.

\section{Tumor xenograft assay}

Female 4-week-old female nude mice were obtained from Slaccas Company. Totally, $2 \times 10^{6}$ A549 cells of each group were injected subcutaneously into either side of mice posterior flank. 4 weeks later, the mice were executed and the size of tumors were measured by caliper for statistical analysis.

\section{Statistical analysis}

The SPSS 17.0 was applied for statistical analysis. The correlations between the expression of Calpain-2 and clinicopathological characteristics were analyzed by the Chi-square test. The OS curves were drawn with the Kaplan-Meier product limit estimator and analyzed with the log-rank test. The correlations between risk factors (related clinicopathological characteristics) and OS were conducted with univariate and multivariate Cox proportional-hazards regression. The risk factors (covariates) included gender, smoking status, differentiation, tumor size, lymph node metastasis, TNM stage and Calpain-2 expression level. The comparison of individual variables were performed with Student's t-test, Chi-square test, Fisher's exact test or Spearman coefficients to analyze the difference between each subgroup in the proper way. Two-tailed tests at $p<0.05$ were considered statistically significant.

\section{Results}

\section{Calpain-2 expression was up-regulated in NSCLC cell lines and tissues}

To explore the Calpain-2 expression in NSCLC and normal cell lines, we performed Western blot and RT-qPCR to analyze the expression difference. In mRNA expression level, Calpain-2 expression was up-regulated significantly in almost all NSCLC cell lines except 95C (Fig. 1A). And in protein expression level, Calpain-2 expression in H1395, A549 and 95D was up-regulated compared with HBE (Fig. 1B). Then, we detected Calpain-2 expression in NSCLC and para-cancerous tissues. As shown in Fig. 1C and 1D, Calpain-2 expression was up-regulated in NSCLC tissues compared with the corresponding paracancerous tissues in both protein and mRNA level. Thus, we could arrive at the conclusion that Calpain-2 expression was up-regulated in NSCLC cell lines and tissues and chose A549 and 95D for further investigation. 
A

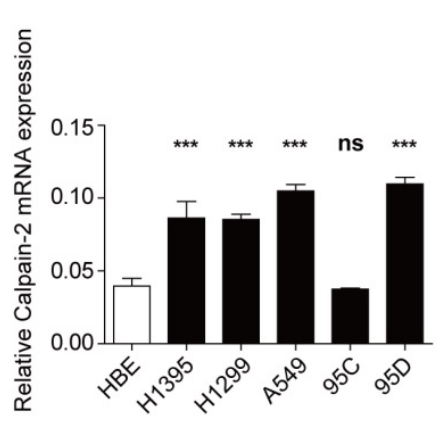

B
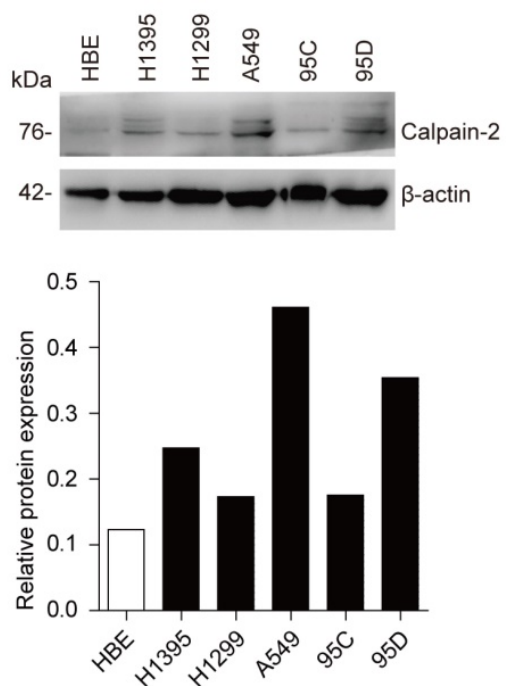

C

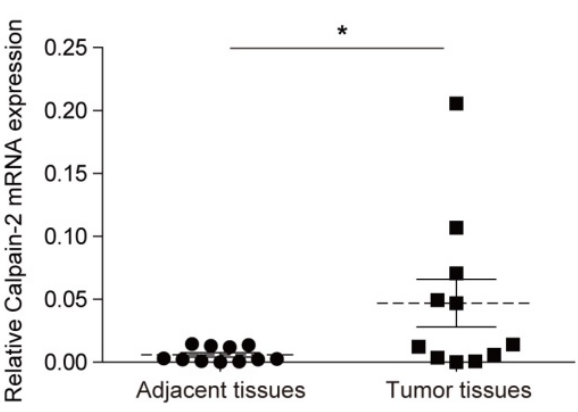

D
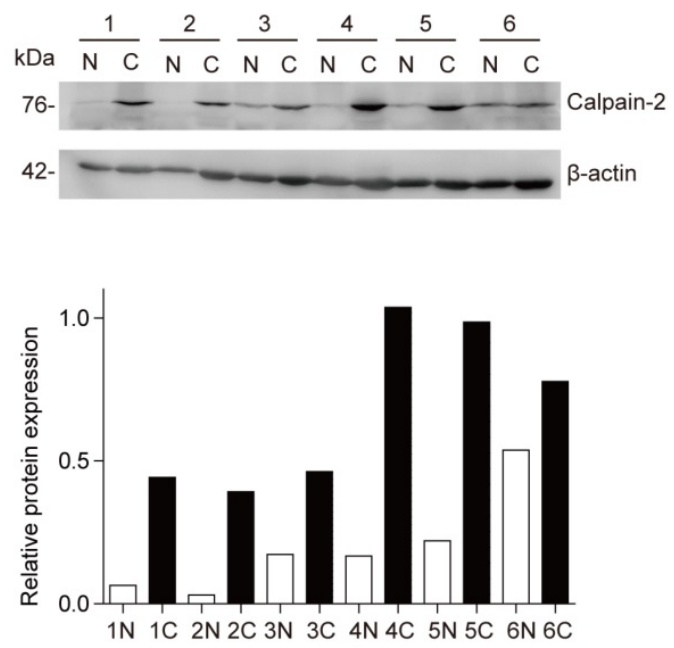

Figure 1. Calpain-2 is up-regulated in NSCLC cell lines and tissues. (A and B) Western blot assay and RT-qPCR were performed to detect the Calpain-2 expression in human normal bronchiolar epithelium cell line (HBE) and NSCLC cell lines. (C and D) Western blot assay and RT-qPCR were performed to detect the Calpain-2 expression in NSCLC tumors and corresponding para-cancerous tissues. "N" stands for "normal para-cancerous tissues" and "C" stands for "cancerous tissues". (ns: no significance, ${ }^{*} \mathrm{p}<0.05,{ }^{*} \mathrm{p}<0.01, * * * \mathrm{p}<0.001$ )

\section{High expression of Calpain-2 was negatively associated with NSCLC patients prognosis}

In a cohort of 208 NSCLC tissues and corresponding adjacent para-cancerous tissues, we performed IHC to detect Calpain-2 expression and tried to analyze the association between Calpain-2 and patients' clinicopathological characteristics. As shown in Fig. 2A, Calpain-2 was expressed mainly in the cytoplasm in cancerous tissues. According to the scoring standard described in the methods and materials, 105 patients were classified into Calpain-2 ${ }^{\text {high }}$ subgroup and 103 patients were classified into Calpain-2 ${ }^{\text {low }}$ subgroup. In the analysis of the association between clinicopathological characteristics and Calpain-2 level, the Chi-square test showed that high Calpain-2 level was positively correlated with differentiation grade significantly (Table 1) (Fig. 2B). In the analysis of OS, the survival curve demonstrated that high Calpain-2 expression correlated with shorter 5-year OS $(p=0.035)$ (Fig. 2C). In univariate analysis of the related factors associated with 5-year OS, lymph node metastasis $(\mathrm{p}<0.001)$, larger tumor size $(\mathrm{p}<0.001)$, higher tumor stage $(\mathrm{p}<0.001)$, and higher Calpain-2 level $(\mathrm{p}=0.001)$ were correlated with shorter 5 -year OS. In multivariate analysis, Calpain-2 $(p=0.043)$, together with tumor size $(p=0.002)$, lymph node metastasis $(p=0.001)$ and tumor stage $(p=0.046)$ were identified as independent prognostic factors for 5-year OS (Table 2).

\section{Calpain-2 promotes the cell proliferation and migration of NSCLC in vitro}

To investigate the bio-function of Calpain-2 on the cell proliferation and migration of NSCLC, we firstly applied 3 pairs of Calpain-2-siRNA sequences to knock down Calpain-2 expression and performed Western blot to test the effect of knockdown. As shown in Fig. S1, 3 pairs of Calpain-2-siRNA all down-regulated the Calpain-2 expression significantly, compared with the Mock and Control subgroups. We chose Calpain-2-siRNA-2 and Calpain-2-siRNA-3 at random for further study. Then, we performed CCK-8 and colony-formation assay and found that the 
proliferation ability of A549 and 95D cell lines were significantly inhibited by the down-regulation of Calpain-2 (Fig. 3A and Fig. S2A). In addition, compared with Control subgroups, the colonyformation assay showed that the numbers of colonies formed by A549 and 95D cell lines were both significantly decreased by down-regulation of Calpain-2 (Fig. 3B). Next, we performed Transwell assay to explore the role of Calpain-2 in cancer cell migration. As shown in Fig. $3 \mathrm{C}$ and Fig. S2B, the migration capability of A549 and 95D cell lines control subgroups were greater than that of the Calpain-2 knock-down subgroups. These data indicated that Calpain-2 promotes the NSCLC cell proliferation and migration of NSCLC in vitro.

\section{Calpain-2 inhibits the cell apoptosis of NSCLC in vitro and promotes the tumorigenesis of NSCLC in vivo}

To explore whether Calpain-2 influenced the cell apoptosis of NSCLC, we performed Annexin V/ PI assay with FACS to detect the percentage of apoptosis in each subgroup. As shown in Fig. 3D and and Fig. S2C, in both A549 and 95D cell lines, the percentages of apoptosis cell were increased after knock-down of Calpain-2, which indicated that Calpain-2 could inhibit the cell apoptosis of NSCLC in vitro.

A

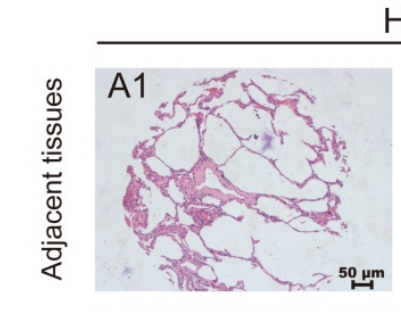

HE
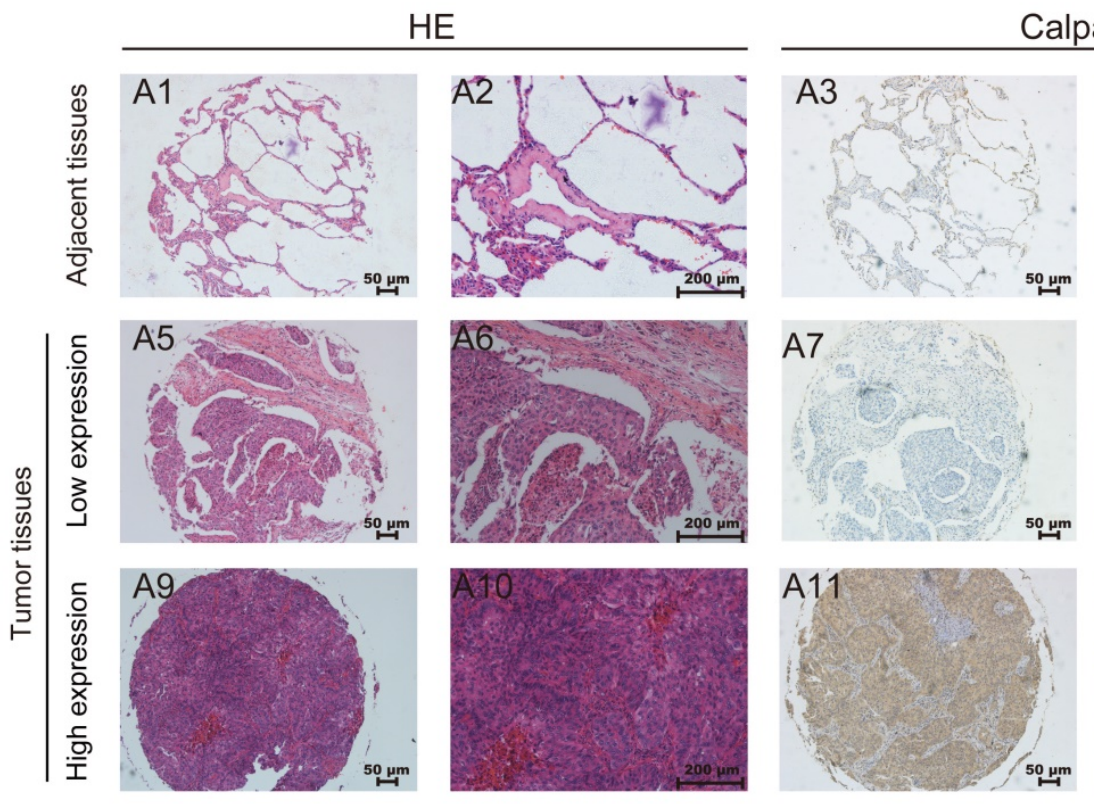

Calpain-2
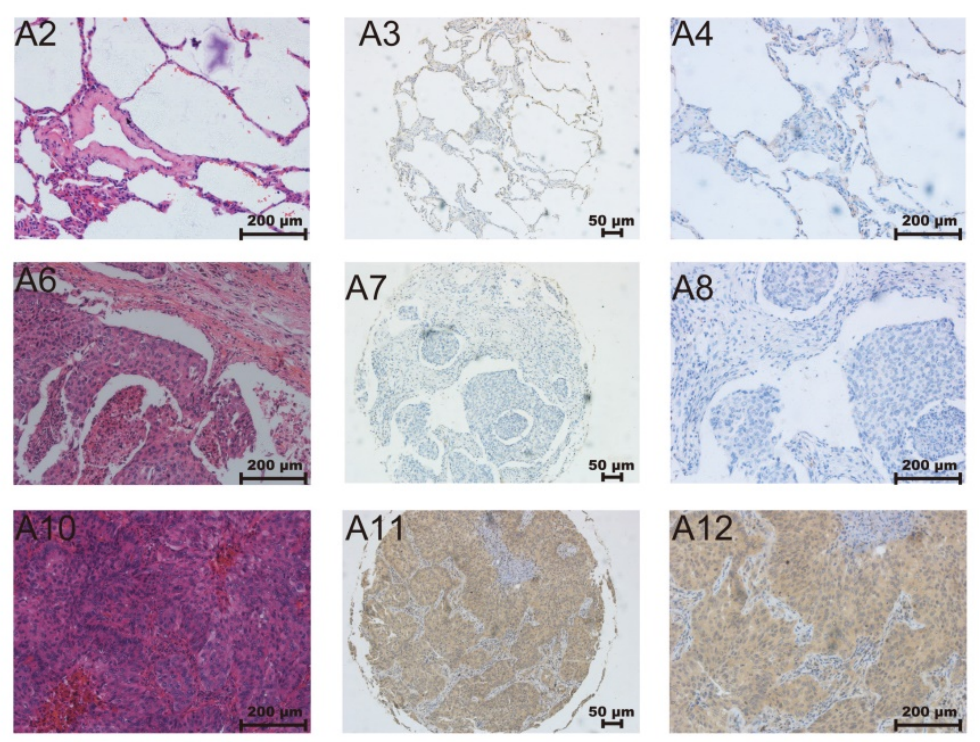

B

C
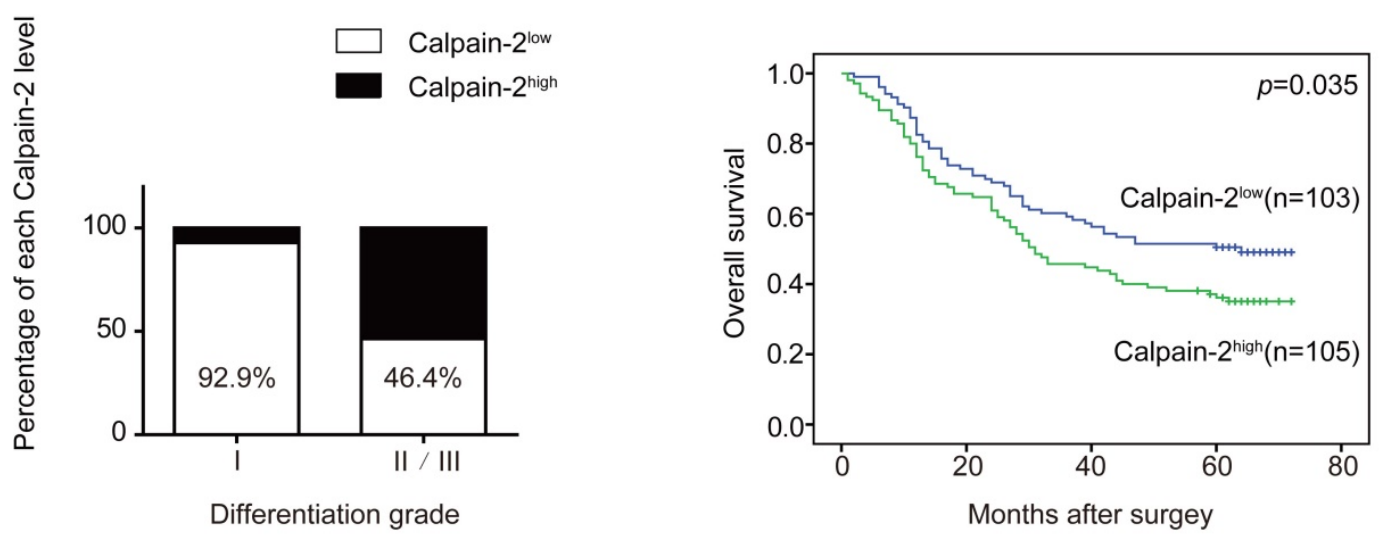

Figure 2. Calpain-2 level is negatively associated with patents' prognosis. (A) IHC analysis of Calpain-2 expression in NSCLC tissues from 208 enrolled patients. The typical images of HE (2 left lines) and Calpain-2 expression (2 right lines) were exhibited. The original magnification $\times 100$ are for $\mathrm{A} 1,3,5,7,9,11$ and original magnification $\times 400$ are for $A 2,4,6,8,10$, 12. (B) The percentage of Calpain-2 expression level in each differentiation grade. (C) OS curves of 208 enrolled NSCLC patients, obtained by Kaplan-Meier product limit estimator, were compared according to Calpain-2 expression level. 
A

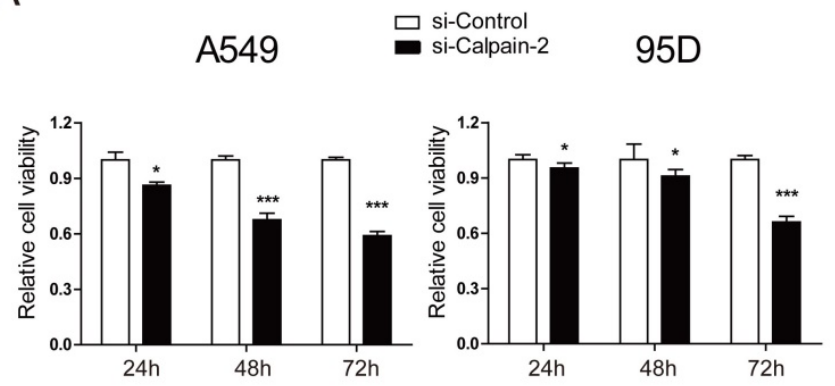

C
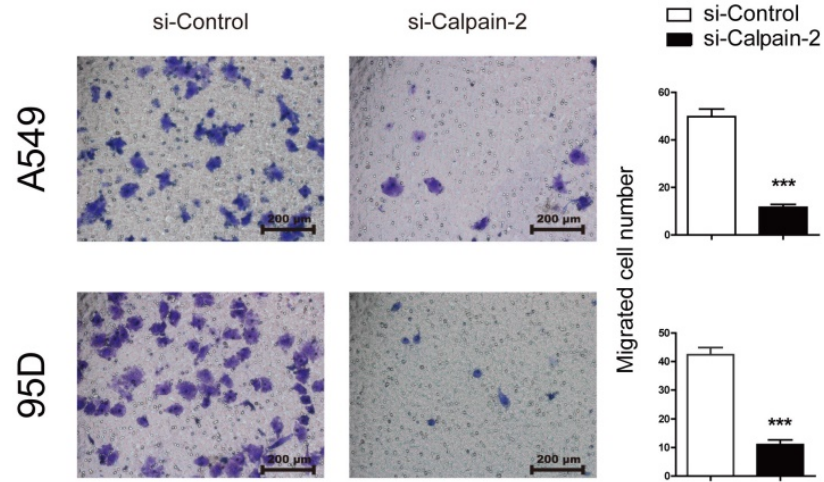

E

A549
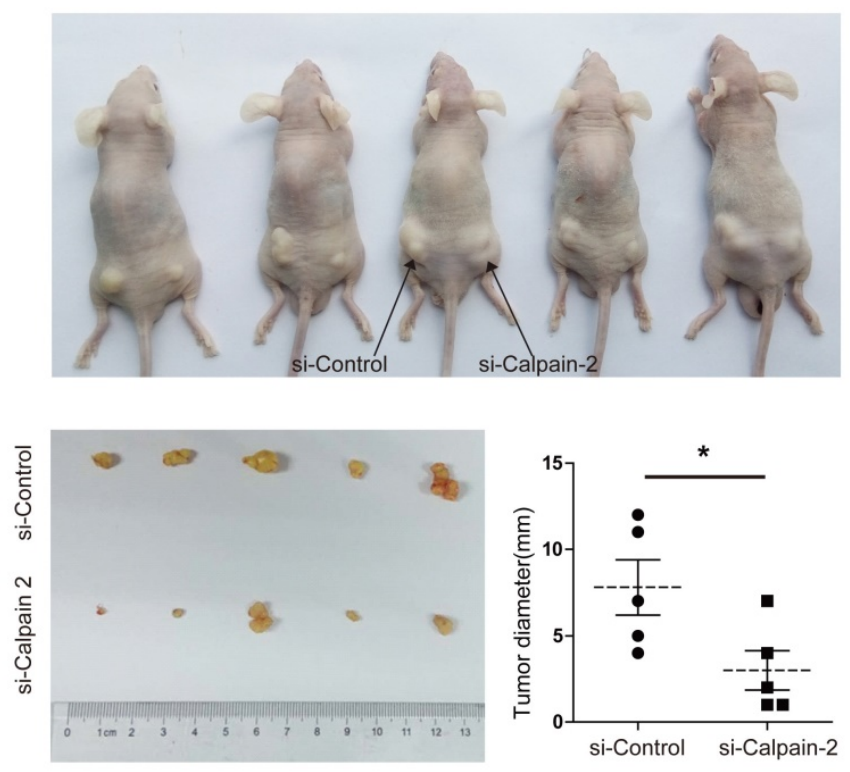

B
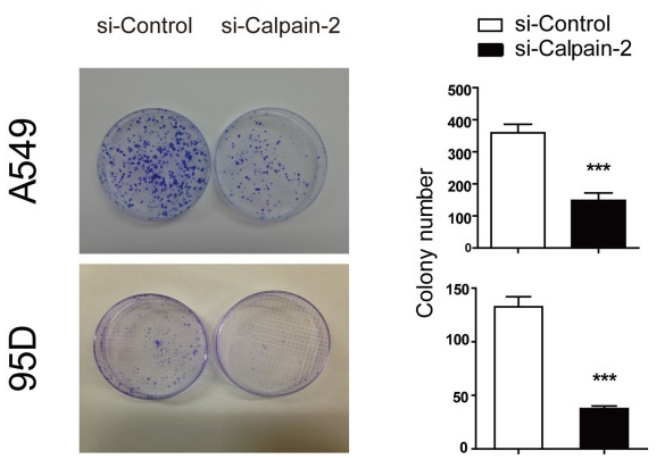

D

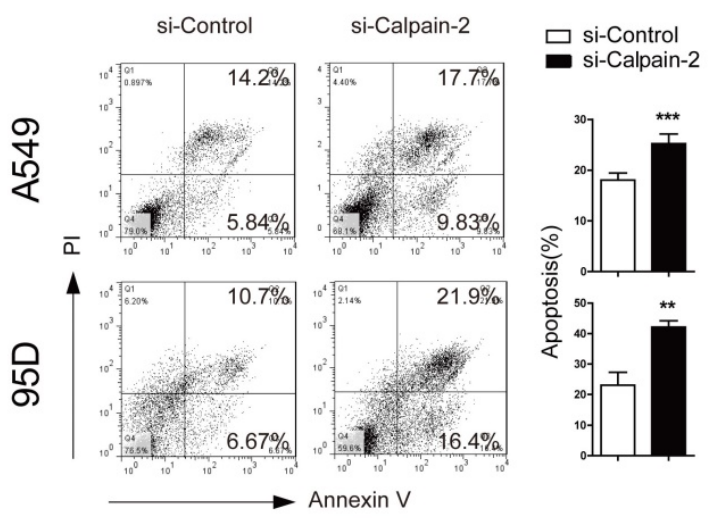

F

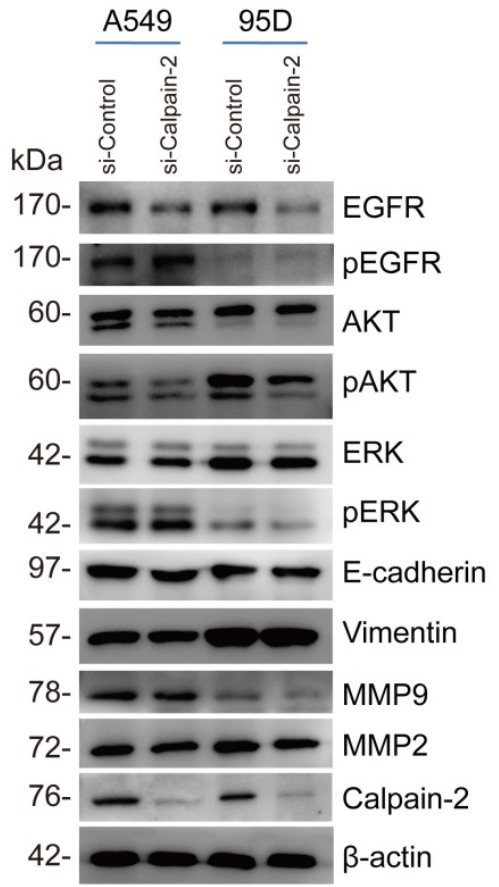

Figure 3. Calpain-2 promotes the progression of NSCLC via the activation of EGFR-pAKT signaling pathway. (A) The proliferative ability was assessed with CCK-8 assay at 24, 48 and 72 hours after transfection. (B) The proliferative ability was assessed with colony-forming assay and analyzed statistically after 2 -week culture. (C) The migration ability was assessed with Transwell assay. (D) The apoptosis of cells was detected with flow cytometry after staining of Annexin $V$ and PI. (E) The proliferative ability was assessed with tumor xenograft assay and analyzed statistically 4 weeks after implantation. (F) Western blot was performed to analyze the expression difference of the related protein after knockdown of Calpain-2. $\beta$-actin was used as an internal reference. (ns: no significance, $*_{p}<0.05$, $* *_{p}<0.01$, $* * * p<$ $0.001)$ 
Table 1. Correlation between Calpain 2 and clinicopathological features in 208 NSCLCs

\begin{tabular}{|c|c|c|c|c|}
\hline \multirow[t]{2}{*}{ Variables } & \multirow{2}{*}{$\begin{array}{l}\text { NO. of } \\
\text { patients }\end{array}$} & \multicolumn{2}{|c|}{ Calpain 2 expression } & \multirow[t]{2}{*}{$P$} \\
\hline & & Low & High & \\
\hline \multicolumn{5}{|l|}{ Age } \\
\hline$<60$ & 102 & $46(45)$ & $56(55)$ & 0.211 \\
\hline$\geq 60$ & 106 & $57(54)$ & $49(46)$ & \\
\hline \multicolumn{5}{|l|}{ Gender } \\
\hline Male & 148 & $70(47)$ & $78(53)$ & 0.314 \\
\hline Female & 60 & $33(55)$ & $27(45)$ & \\
\hline \multicolumn{5}{|l|}{ Smoking status } \\
\hline Smokers & 84 & $44(53)$ & $40(48)$ & 0.497 \\
\hline Non-smokers & 124 & $59(48)$ & $65(52)$ & \\
\hline \multicolumn{5}{|l|}{ Histological type } \\
\hline squamous cell carcinoma & 85 & $35(41)$ & $50(49)$ & 0.077 \\
\hline adenocarcinomas & 110 & $59(54)$ & $51(46)$ & \\
\hline Other\# & 13 & $9(69)$ & $4(31)$ & \\
\hline \multicolumn{5}{|l|}{ Tumor stage } \\
\hline I - II & 144 & $67(46)$ & $77(54)$ & 0.196 \\
\hline III-IV & 64 & $36(56)$ & $38(44)$ & \\
\hline \multicolumn{5}{|l|}{ Lymph node metastasis } \\
\hline Yes & 90 & $44(49)$ & $46(51)$ & 0.874 \\
\hline No & 118 & $59(50)$ & $59(50)$ & \\
\hline \multicolumn{5}{|l|}{ Tumor size } \\
\hline$<3 \mathrm{~cm}$ & 69 & $34(49)$ & $35(51)$ & 0.960 \\
\hline$\geq 3 \mathrm{~cm}$ & 139 & $69(50)$ & $70(50)$ & \\
\hline \multicolumn{5}{|l|}{ Differentiation } \\
\hline Well & 14 & 13(93) & $1(7)$ & 0.001 \\
\hline moderate/Poor & 194 & $90(46)$ & $104(54)$ & \\
\hline
\end{tabular}

To investigate the role of Calpain-2 in tumorigenesis in vivo, we performed tumor xenograft assay and demonstrated that A549 Control subgroup could form significantly larger tumors than the Calpain-2 knock-down subgroup did, which indicated that Calpain-2 played a potential role in promoting cell tumorigenesis of NSCLC (Fig. 3E).

\section{Calpain-2 promotes the progression of NSCLC possibly through the activation of EGFR-pAKT signaling pathway}

It has been reported that Calpain-2 could activate Akt in pulmonary artery smooth muscle cells [16]. We tried to explore whether similar mechanism existed in the role of Calpain-2 in promoting the progression of NSCLC. We performed Western blot assay to detect some classical signaling pathway gene, including EGFR and ERK which are related to AKT. As shown in Fig. 3F, after knock-down of Calpain-2, the pAKT and EGFR expression decreased, while the AKT, pEGFR, ERK and pERK showed no significant difference. Meanwhile, we detected some other classical signaling pathway related to tumor progression. The data showed that knock-down of Calpain-2 made no difference in the expression of EMT markers (E-cadherin and Vimentin) and MMP family (MMP-2 and MMP-9). We speculated that Calpain-2 might activate EGFR-pAKT signaling pathway to promote the progression of NSCLC.

\section{Calpain-2 enhanced NSCLC cells resistance to PTX possibly via the activation EGFR.}

EGFR has been reported to promote chemotherapy resistance, through the activation of multiple downstream pathways, including NF-kB, PI3K/Akt, etc. $[17,18]$. Additionally, Calpain-2 has also been reported to be associated with response to platinum based chemotherapy in ovarian cancer [11]. Therefore, we speculated whether Calpain-2 could enhance NSCLC cells resistance to PTX possibly via the activation EGFR. We performed CCK-8 to detect the function of PTX on cell viability and found that A549 and 95D cells viability were reduced in a PTX-dose-dependent manner (Fig. 4A). The IC50 value of A549 and 95D cells was $53.06 \pm 5.26 \mathrm{nM}$ and $44.60 \pm 8.02 \mathrm{nM}$, respectively. Then, we knocked down Calpain-2 expression in both two cell lines to analyze the function of Calpain-2 PTX resistance by CCK-8. As shown in Fig. 4B, knock-down of Calpain-2 enhanced PTX-mediated suppression of A549 and 95D cells viability, most notably at lower doses of PTX $(10-20 \mathrm{nM})$. We further detect the apoptosis difference and found that knock-down of Calpain-2 increased PTX-mediated apoptosis of A549 and 95D cells (Fig. $4 \mathrm{C})$. These data above indicated that Calpain-2 could enhance NSCLC cells resistance to PTX.

To prove the mechanism under the phenomena, we performed Western blot to detect EGFR and other related gene expressional difference led by the treatment of siRNA-Calpain-2, PTX, or both of them. As shown in Fig. 4D, in both two cell lines, PTX treatment down-regulated Calpain-2, EGFR and pAKT protein level in the comparison of siRNAControl ${ }^{\mathrm{PTX}(-)}$ vs. siRNA-Control ${ }^{\mathrm{PTX}(+)}$ subgroups and siRNA-Calpain-2 ${ }^{\text {PTX(-) }}$ vs. siRNA-Calpain-2 ${ }^{\text {PTX(+) }}$ subgroups. And the subgroups treated with both knockdown of Calpain-2 and PTX decreased Calpain2, EGFR and pAKT protein level most significantly compared with the other three subgroups. However, PTX treatment made no significant difference in the protein level of pEGFR, AKT, ERK and pERK between siRNA-Control ${ }^{\text {PTX }}$ subgroup and siRNA-Calpain-2 ${ }^{\text {PTX }}$ subgroup. Therefore, both knock-down of Calpain-2 and treat with PTX reduced levels of EGFR and pAKT and cell proliferation, but increased cell apoptosis. These results implied that Calpain- 2 could enhance NSCLC cells resistance to PTX possibly via the activation EGFR-pAKT signaling pathway.

\section{Discussion}

The Calpain family has been studied in its role in cancer progression and response to therapy in different types of tumors [19-21]. However, the 
bio-function of Calpain-2 on NSCLC still remained unclear. In this study, we verified that Calpain-2 expression was up-regulated in NSCLC tissues and cell lines. Through the analysis of survival, we demonstrated that high Calpain-2 expression correlated with high differentiation grade and poor 5 -year survival, serving as an independent prognostic predictor. In the study of bio-function of Calpain-2, the data showed that Calpain-2 promoted the cell proliferation and migration, while inhibited apoptosis ability of NSCLC, and enhance the resistance to PTX possibly via EGFR-pAKT signaling pathway. These results suggested that Calpain- 2 might serve as a novel therapeutic target to prevent or postpone the progression of NSCLC.

Table 2. Univariate and Multivariate analysis of factors associated with OS

\begin{tabular}{|c|c|c|c|c|c|c|}
\hline \multirow[t]{2}{*}{ Variables } & \multicolumn{3}{|c|}{ Univariate Analysis } & \multicolumn{3}{|c|}{ Multivariate Analysis } \\
\hline & HR & $95 \% \mathrm{CI}$ & $P$ & HR & $95 \% \mathrm{CI}$ & $P$ \\
\hline Gender (female vs. male) & 0.789 & $0.526-1.183$ & 0.251 & & & \\
\hline Smoking status (non-smokers vs. smokers) & 0.779 & $0.543-1.118$ & 0.175 & & & \\
\hline Differentiation(Poor/Moderate vs. Well) & 3.889 & $1.235-12.24$ & 0.020 & 0.454 & $0.141-1.463$ & 0.186 \\
\hline Lymph node metastasis(Yes vs. No) & 3.042 & 2.103-4.399 & $<0.001$ & 0.482 & $0.311-0.748$ & 0.001 \\
\hline Tumor size $(\geq 3 \mathrm{~cm}$ vs. $<3 \mathrm{~cm})$ & 2.755 & $1.758-4.318$ & $<0.001$ & 0.480 & $0.302-0.763$ & 0.002 \\
\hline Tumor stage (III-IV vs. I-II) & 2.771 & $1.922-3.993$ & $<0.001$ & 0.640 & $0.413-0.992$ & 0.046 \\
\hline Calpain 2 level(high vs. low) & 1.857 & $1.280-2.694$ & 0.001 & 0.684 & $0.474-0.988$ & 0.043 \\
\hline
\end{tabular}

Abbreviations: OS, overall survival; $95 \% \mathrm{CI}$, 95\% confidence interval; $\mathrm{HR}$, hazard ratio.

A

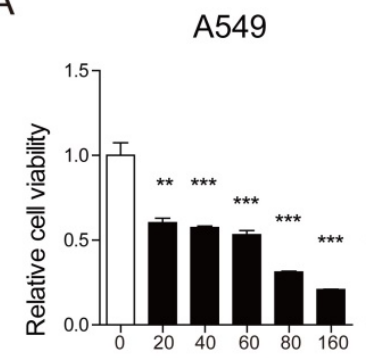

$\operatorname{PTX}(\mathrm{nM})$

C

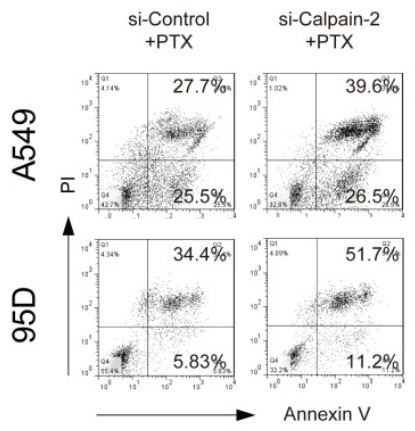

E
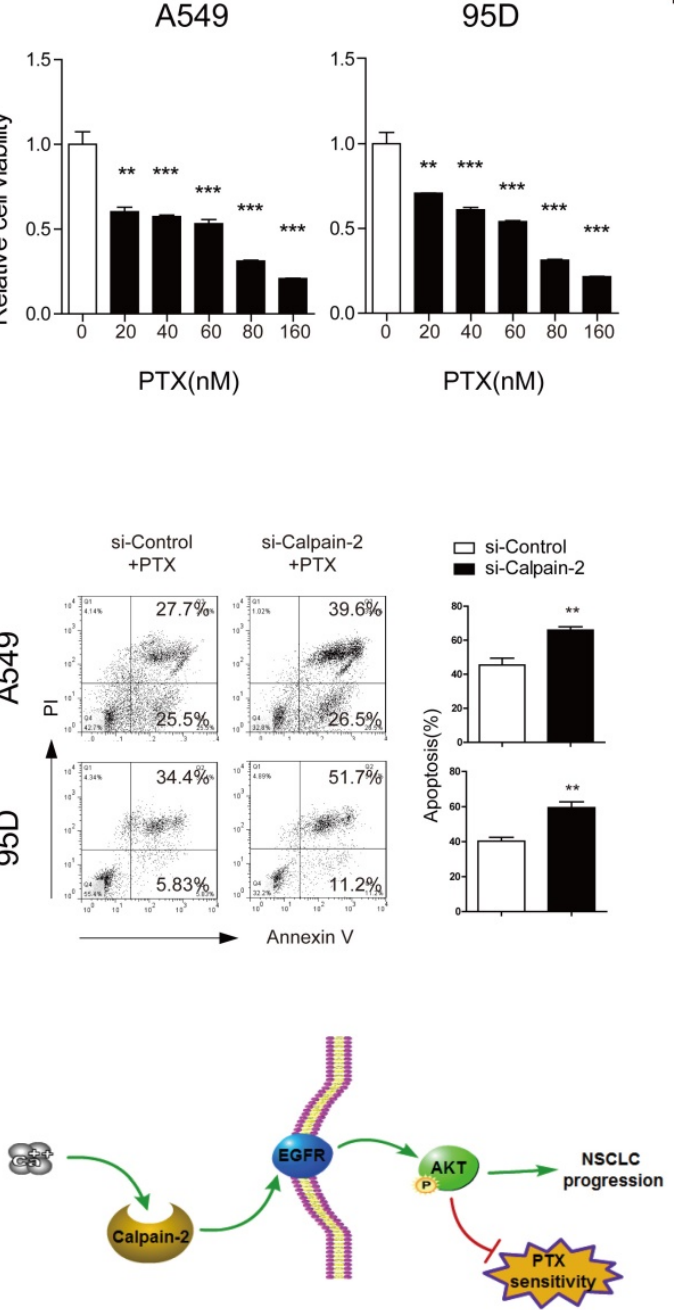

B

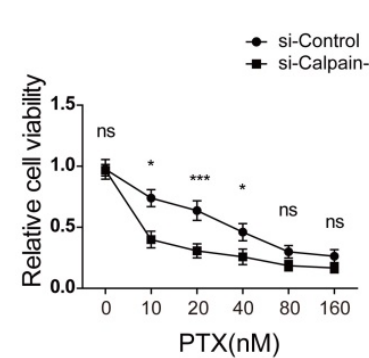

95D

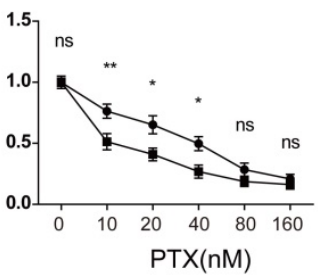

$\mathrm{D}$

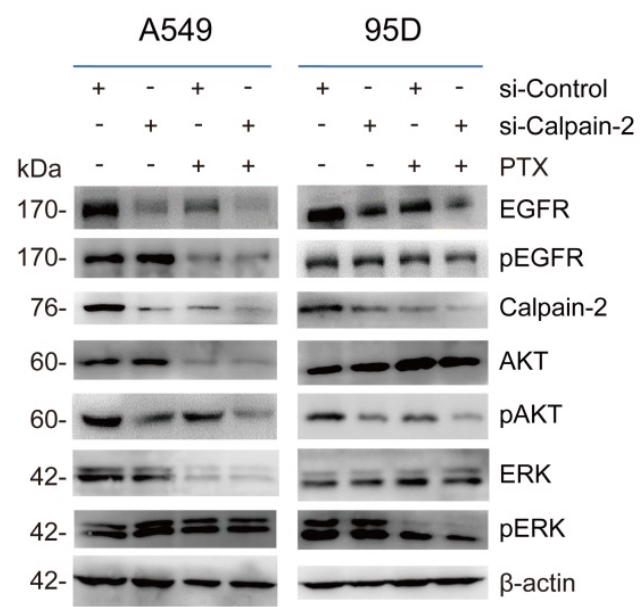

Figure 4. Calpain-2 promotes the chemoresistance to paclitaxel. (A) The cells were treated with different doses of PTX (0, 10, $20,40,80$ and $160 \mathrm{nM}$ ) for $48 \mathrm{~h}$. The IC50 value of A549 and 95D cells was $53.06 \pm 5.26 \mathrm{nM}$ and $44.60 \pm 8.02 \mathrm{nM}$, respectively. (B and C) The cells transfected with si-control or si-Calpain-2 were treated with PTX for $24 \mathrm{~h}$. Cells viability and apoptosis were evaluated by the CCK-8 and Annexin V/ PI assays. (D) Western blot was performed to analyze the difference of related protein expression level after knockdown of Calpain-2 and treatment of PTX. $\beta$-actin was used as an internal reference. (E) Graph summarized the mechanism for Calpain-2 to promote the progression of NSCLC and chemoresistance to PTX. (ns: no significance, ${ }^{*} \mathrm{p}<0.05, * * \mathrm{p}<0.01, * * * \mathrm{p}<0.001$ ) 
Calpain-2, as the large subunit of $\mathrm{m}$-Calpain, has been reported to up-regulate in multiple tumors and play a crucial role in promoting cancer cells proliferation and migration, but inhibiting apoptosis ability. Li and colleagues demonstrated that silencing Calpain-2 could inhibit prostate cancer cells proliferation and migration [22]. In mammary carcinoma, Ho and colleagues also verified that depletion of Calpain-2 suppressed cells proliferation and migration [23]. By the use of Calpain inhibitor II, acute lymphoblastic leukemia and non-Hodgkin's lymphoma cells apoptosis could be triggered significantly [24]. Consistent to these results, our data showed that Calpain-2 was overexpressed in NSCLC, and knockdown of Calpain-2 could inhibit proliferation and migration, and promote apoptosis, which acted together to promote the progression of NSCLC.

To seek underlying mechanism under the bio-function of Calpain-2, Jang and colleagues proved that calpain-2 is required for glioblastoma cell invasion through the regulation of MMP2 [8]. Abeyrathna and colleagues demonstrated that Calpain-2 activates Akt via TGF- $\beta 1$-mTORC2 pathway in pulmonary artery smooth muscle cells [16]. Similarly, Calpain-2 was also reported to mediate $\mathrm{AKT} / \mathrm{mTOR}$ signal pathway in prostate cancer [22]. According to the accumulated data above, we tried to detect whether Calpain-2 could activated some classical signaling pathways, including: EMT (epithelial-mesenchymal transition), MMP, EGFRAKT/ERK pathway. Our results showed that Calpain-2 could up-regulated pAKT and EGFR level, but made no significant difference in AKT, pEGFR, ERK and pERK level. Some other reports about EGFR-AKT signaling pathway also showed that downstream AKT activation was inhibited after decreasing total EGFR [25]. Phosphorylation is required for EGFR signaling. Mostly, phosphorylation of EGFR is also decreased when the total EGFR protein is decreased. However, knock-down of Calpain-2 didn't reduce pEGFR level at Y1068, one of the most frequent sites of EGFR phosphorylation. For possible reasons, as previously reported, there exists other phosphorylated sites in EGFR protein, such as Y992, Y1045, Y1148, Y1173, and even some unknown sites [26]. These sites may function under different conditions [27, 28]. In the regulation of Calpain-2, other sites rather than Y1068 may be involved in. Therefore, we speculated that Calpain-2 might activate EGFR-pAKT pathway to promote the progression of NSCLC. In addition to Calpain-2, CAPN4 has been proved to promote NSCLC through the activation of MMP-2 signaling pathway in our previous study [6]. Tan and colleagues have found that Calpain-1, the large catalytic subunit of $\mu$-Calpain, could faciliate EMT in TGF- $\beta 1$-treated lung cancer cells through the activation of PI3K/AKT signaling pathway [29]. In this study, the Western blot assay showed that the MMP-2, E-cadherin and Vimentin expression level showed no difference after knockdown of Calpain-2. Since the progression of tumor was a consequence of multiple risk factors [30], the combination of Calpain-2 and CAPN4, function as m-Calpain, might produce synergies, together with $\mu$-Calpain, through the activation of multiple signaling pathway, to facilitate the progression of NSCLC.

PTX is one of the most applied first-line chemotherapy options for NSCLC [31]. However, the therapeutic efficacy of PTX might be gradually hampered by chemotherapeutic resistance with the passage of time, which became a major adverse factor for patients' prognosis [32]. Mounting evidences proved that EGFR downstream pathways, including PI3K/Akt, STAT and the NF-kB signaling pathways are involved in the induction of chemoresistance [33-35]. Li and colleagues demonstrated that the down-regulation of membrane EGFR could be a potential anti-cancer way to enhance PTX treatment efficacy [36]. Therefore, we tried to explore whether Calpain-2 could enhance the chemoresistance of NSCLC cells to PTX through the activation of EGFR. Our data demonstrated that knockdown of Calpain-2 led to high tendency to apoptosis induced by PTX. And the treatment of PTX also decreased Calpain-2 expression level. Meanwhile, knockdown of Calpain-2 enhanced the paclitaxel-mediated downregulation of EGFR and its downstream pAKT level, which was in accordance to $\mathrm{Li}^{\prime}$ 's results [36] and implied the stimulating role of Calpain-2 in the chemoresistance to PTX, through the activation of EGFR/pAKT signaling pathway.

In addition to the chemoresistance caused by Calpain-2 through the activation of EGFR signaling pathway, the tyrosine kinase inhibitor (TKI) has been extensively proved to be quite effective to the NSCLC patients with some types of EGFR mutation in clinical [37]. Despite this, it is inevitable to arouse the resistance to TKI with the long-term application of TKI. The T790M mutation has been proved to be one of the frequent factors leading to the resistance to TKI [38]. However, there still exists some mutation, including MET amplification, transformation to SCLC, PIK3CA mutations, even some unknown mutation lack of targeted-therapy, resulting in the failure of therapy [39]. Therefore, whether targeted inhibition of Calpain-2 could contribute to the TKI therapy for NSCLC might be a potential therapeutic strategy and needs further investigation, which is of 
high clinical value.

To summarize, we have demonstrated that Calpain-2 expression is up-regulated in NSCLC cell lines and tissues. Meanwhile, Calpain-2 serves as an independent prognostic factor. In bio-function, Calpain-2 plays a crucial role in promoting NSCLC cells proliferation and migration, but inhibiting apoptosis ability possibly via the activation of EGFR-pAKT signaling pathway, through which Calpain-2 could enhance the chemoresistance to PTX. Thus, Calpain-2 might offer a novel therapeutic strategy to prevent or postpone the progression of NSCLC.

\section{Abbreviations}

NSCLC: non-small cell lung cancer; OS: overall survival; PI: Propidium Iodide; HR: hazard ratio; $\mathrm{CI}$ : confidence interval; TMN: tumor-node-metastasis; PTX: paclitaxel; TKI: tyrosine kinase inhibitor.

\section{Supplementary Material}

Supplementary figures and tables. http://www.ijbs.com/v15p0127s1.pdf

\section{Acknowledgements}

We appreciated all the supports during our study very much, including the National Natural Science Foundation (81372313 and 81401876), the Doctoral Fund for Young scholar of Ministry of Education of China (20110071120065), the Doctoral Fund for new teacher, Personnel Training Plan of Zhongshan Hospital, Fudan University and Outstanding study plan of Fudan University.

\section{Competing Interests}

The authors have declared that no competing interest exists.

\section{References}

1. Torre LA, Bray F, Siegel RL, Ferlay J, Lortet-Tieulent J, Jemal A. Global cancer statistics, 2012. CA: a cancer journal for clinicians. 2015; 65: 87-108.

2. Lin JJ, Cardarella S, Lydon CA, Dahlberg SE, Jackman DM, Janne PA, et al. Five-Year Survival in EGFR-Mutant Metastatic Lung Adenocarcinoma Treated with EGFR-TKIs. Journal of thoracic oncology : official publication of the International Association for the Study of Lung Cancer. 2016; 11: 556-65.

3. Siegel RL, Miller KD, Jemal A. Cancer Statistics, 2017. CA: a cancer journal for clinicians. 2017; 67: 7-30.

4. Sorimachi H, Ishiura S, Suzuki K. Structure and physiological function of calpains. The Biochemical journal. 1997; 328 ( Pt 3): 721-32.

5. Goll DE, Thompson VF, Li H, Wei W, Cong J. The calpain system. Physiological reviews. 2003; 83: 731-801.

6. Gu J, Xu FK, Zhao GY, Lu CL, Lin ZW, Ding JY, et al. Capn4 promotes non-small cell lung cancer progression via upregulation of matrix metalloproteinase 2. Medical oncology. 2015; 32: 51

7. Lakshmikuttyamma A, Selvakumar P, Kanthan R, Kanthan SC, Sharma RK. Overexpression of $\mathrm{m}$-calpain in human colorectal adenocarcinomas. Cancer epidemiology, biomarkers \& prevention : a publication of the American Association for Cancer Research, cosponsored by the American Society of Preventive Oncology. 2004; 13: 1604-9.

8. Jang HS, Lal S, Greenwood JA. Calpain 2 is required for glioblastoma cell invasion: regulation of matrix metalloproteinase 2 . Neurochemical research. 2010; 35: 1796-804

9. Mamoune A, Luo JH, Lauffenburger DA, Wells A. Calpain-2 as a target for limiting prostate cancer invasion. Cancer research. 2003; 63: 4632-40.
10. Miao C, Liang C, Tian Y, Xu A, Zhu J, Zhao K, et al. Overexpression of CAPN2 promotes cell metastasis and proliferation via AKT/mTOR signaling in renal cell carcinoma. Oncotarget. 2017; 8: 97811-21.

11. Storr SI, Safuan S, Woolston CM, Abdel-Fatah T, Deen S, Chan SY, et al. Calpain-2 expression is associated with response to platinum based chemotherapy, progression-free and overall survival in ovarian cancer. Journal of cellular and molecular medicine. 2012; 16: 2422-8.

12. Pease-Raissi SE, Pazyra-Murphy MF, Li Y, Wachter F, Fukuda Y, Fenstermacher SJ, et al. Paclitaxel Reduces Axonal Bclw to Initiate IP3R1-Dependent Axon Degeneration. Neuron. 2017; 96(e6): 373-86.

13. Gu J, Ding JY, Lu CL, Lin ZW, Chu YW, Zhao GY, et al. Overexpression of CD88 predicts poor prognosis in non-small-cell lung cancer. Lung cancer. 2013; 81: 259-65.

14. Zhao GY, Lin ZW, Lu CL, Gu J, Yuan YF, Xu FK, et al. USP7 overexpression predicts a poor prognosis in lung squamous cell carcinoma and large cell carcinoma. Tumour biology : the journal of the International Society for Oncodevelopmental Biology and Medicine. 2015; 36: 1721-9.

15. Zhao GY, Ding JY, Lu CL, Lin ZW, Guo J. The overexpression of 14-3-3zeta and Hsp27 promotes non-small cell lung cancer progression. Cancer. 2014; 120: $652-63$.

16. Abeyrathna $\mathrm{P}$, Kovacs $\mathrm{L}$, Han $\mathrm{W}, \mathrm{Su}$ Y. Calpain-2 activates Akt via TGF-beta1-mTORC2 pathway in pulmonary artery smooth muscle cells. American journal of physiology Cell physiology. 2016; 311: C24-34.

17. Tanaka K, Babic I, Nathanson D, Akhavan D, Guo D, Gini B, et al. Oncogenic EGFR signaling activates an mTORC2-NF-kappaB pathway that promotes chemotherapy resistance. Cancer discovery. 2011; 1: 524-38.

18. Petitprez A, Larsen AK. Irinotecan resistance is accompanied by upregulation of EGFR and Src signaling in human cancer models. Current pharmaceutical design. 2013; 19: 958-64.

19. Storr SJ, Lee KW, Woolston CM, Safuan S, Green AR, Macmillan RD, et al. Calpain system protein expression in basal-like and triple-negative invasive breast cancer. Annals of oncology : official journal of the European Society for Medical Oncology. 2012; 23: 2289-96.

20. Chen B, Tang J, Guo YS, Li Y, Chen ZN, Jiang JL. Calpains are required for invasive and metastatic potentials of human HCC cells. Cell biology international. 2013; 37: 643-52.

21. Cortesio CL, Chan KT, Perrin BJ, Burton NO, Zhang S, Zhang ZY, et al. Calpain 2 and PTP1B function in a novel pathway with $\mathrm{Src}$ to regulate invadopodia dynamics and breast cancer cell invasion. The Journal of cell biology. 2008; 180: 957-71.

22. Li P, Miao C, Liang C, Shao P, Wang Z, Li J. Silencing CAPN2 Expression Inhibited Castration-Resistant Prostate Cancer Cells Proliferation and Invasion via $\mathrm{AKT} / \mathrm{mTOR}$ Signal Pathway. BioMed research international. 2017; 2017: 2593674 .

23. Ho WC, Pikor L, Gao $Y$, Elliott BE, Greer PA. Calpain 2 regulates Akt-FoxO-p27(Kip1) protein signaling pathway in mammary carcinoma. The Journal of biological chemistry. 2012; 287: 15458-65.

24. Zhu DM, Uckun FM. Calpain inhibitor II induces caspase-dependent apoptosis in human acute lymphoblastic leukemia and non-Hodgkin's lymphoma cells as well as some solid tumor cells. Clinical cancer research : an official journal of the American Association for Cancer Research. 2000; 6: 2456-63.

25. Jang JW, Song Y, Kim SH, Kim JS, Kim KM, Choi EK, et al. CD133 confers cancer stem-like cell properties by stabilizing EGFR-AKT signaling in hepatocellular carcinoma. Cancer letters. 2017; 389: 1-10.

26. Nyati MK, Morgan MA, Feng FY, Lawrence TS. Integration of EGFR inhibitors with radiochemotherapy. Nature reviews Cancer. 2006; 6: 876-85.

27. Sebastian A, Pandey V, Mohan CD, Chia YT, Rangappa S, Mathai J, et al. Novel Adamantanyl-Based Thiadiazolyl Pyrazoles Targeting EGFR in Triple-Negative Breast Cancer. ACS omega. 2016; 1: 1412-24.

28. Tong J, Taylor P, Peterman SM, Prakash A, Moran MF. Epidermal growth factor receptor phosphorylation sites Ser991 and Tyr998 are implicated in the regulation of receptor endocytosis and phosphorylations at Ser1039 and Thr1041. Molecular \& cellular proteomics : MCP. 2009; 8: 2131-44.

29. Tan WJ, Tan OY, Wang T, Lian M, Zhang L, Cheng ZS. Calpain 1 regulates TGF-beta1-induced epithelial-mesenchymal transition in human lung epithelial cells via PI3K/Akt signaling pathway. American journal of translational research. 2017; 9: 1402-9.

30. Maranchie JK. Epigenetic factors affect tumor initiation, progression and recurrence. The Journal of urology. 2014; 192: 1318-9.

31. Ettinger DS, Wood DE, Aisner DL, Akerley W, Bauman J, Chirieac LR, et al. Non-Small Cell Lung Cancer, Version 5.2017, NCCN Clinical Practice Guidelines in Oncology. Journal of the National Comprehensive Cancer Network : JNCCN. 2017; 15: 504-35.

32. Liu R, Liu X, Zheng $\mathrm{Y}, \mathrm{Gu}$ J, Xiong $\mathrm{S}$, Jiang $\mathrm{P}$, et al. MicroRNA-7 sensitizes non-small cell lung cancer cells to paclitaxel. Oncology letters. 2014; 8: 2193-200.

33. Wilks ST. Potential of overcoming resistance to HER2-targeted therapies through the PI3K/Akt/mTOR pathway. Breast. 2015; 24: 548-55.

34. Walker SR, Nelson EA, Zou L, Chaudhury M, Signoretti S, Richardson A, et al. Reciprocal effects of STAT5 and STAT3 in breast cancer. Molecular cancer research : MCR. 2009; 7: 966-76.

35. Pushkarev VV, Starenki DV, Pushkarev VM, Kovzun OI, Tronko MD. Inhibitor of the transcription factor NF-kappaB, Dhmeq, enhances the effect of 
paclitaxel on cells of anaplastic thyroid carcinoma in vitro and in vivo. Ukrainian biochemical journal. 2015; 87: 63-74.

36. Li H, Duan ZW, Xie P, Liu YR, Wang WC, Dou SX, et al. Effects of paclitaxel on EGFR endocytic trafficking revealed using quantum dot tracking in single cells. PloS one. 2012; 7: e45465.

37. Furuyama K, Harada T, Iwama E, Shiraishi $Y$, Okamura K, Ijichi K, et al. Sensitivity and kinase activity of epidermal growth factor receptor (EGFR) exon 19 and others to EGFR-tyrosine kinase inhibitors. Cancer science. 2013; 104: 584-9.

38. Denis MG, Vallee A, Theoleyre S. EGFR T790M resistance mutation in non small-cell lung carcinoma. Clinica chimica acta; international journal of clinical chemistry. 2015; 444: 81-5.

39. Sequist LV, Waltman BA, Dias-Santagata D, Digumarthy S, Turke AB, Fidias $\mathrm{P}$, et al. Genotypic and histological evolution of lung cancers acquiring resistance to EGFR inhibitors. Science translational medicine. 2011; 3: 75ra26. 